\title{
Alternative bei allergischer Rhinitis
}

\author{
Bei der Auslösung der allergischen Rhinitis spielen neben Histamin \\ auch Cysteinyl-Leukotriene wie LTC4 und LTD4 eine wichtige Rolle. \\ Eine große kontrollierte klinische Studie untersuchte die Wirksam- \\ keit einer Monotherapie mit dem Leukotrien-Rezeptorantagonisten \\ Montelukast bei saisonaler allergischer Rhinitis.
}

\begin{abstract}
eukotriene wie LTC4 und LTD4 - werden durch Entzündungszellen wie Mastzellen und Eosinophile synthetisiert und freigesetzt. Sie tragen zur Symptomatik bei der allergischen Rhinitis und auch beim allergischen Asthma bronchiale bei. Die klinische Wirksamkeit von Leukotrien-Rezeptorantagonisten beim Asthma im Kindes- und Erwachsenenalter ist gut dokumentiert. Zum Nachweis der Effektivität dieser Rezeptorenblocker bei akuten saisonalen nasalen Allergien wurden 1.302 Patienten im Alter zwischen 15 und 81 Jahren randomisiert mit $10 \mathrm{mg}$ Montelukast $(\mathrm{n}=348), 10 \mathrm{mg}$ Loratadin $(n=602)$ oder Plazebo $(n=352)$ behandelt. Die Medikation wurde jeweils zur Nacht kontinuierlich über 14 Tage während des Frühjahrs gegeben.
\end{abstract}

Die beiden Verumtherapeutika Montelukast und Loratadin erzielten mit jeweils $\mathrm{p} \leq 0,001$ signifikant bessere Symptomreduktionen als Plazebo bei den primären Zielkriterien nasale Kongestion, Rhinorrhö, Juckreiz und Niesen während des Tages, gemessen in einer Intensitätsskala von 0 bis 3 . Entsprechend verhielten sich Symptome wie z. B. die Symptomatik zur Nacht oder an den Augen. Auch der GesamtScore der krankheitsbezogenen Lebensqualität (Rhinokonjunktivitis-Lebensqualitätsfragebogen nach Juniper) war unter Montelukast oder dem Antihistaminikum vergleichbar besser als unter Plazebo. Die Nebenwirkungsrate der Verummedikationen lag auf Plazeboniveau: In allen drei Gruppen wurden am häufigsten Kopfschmerzen genannt.

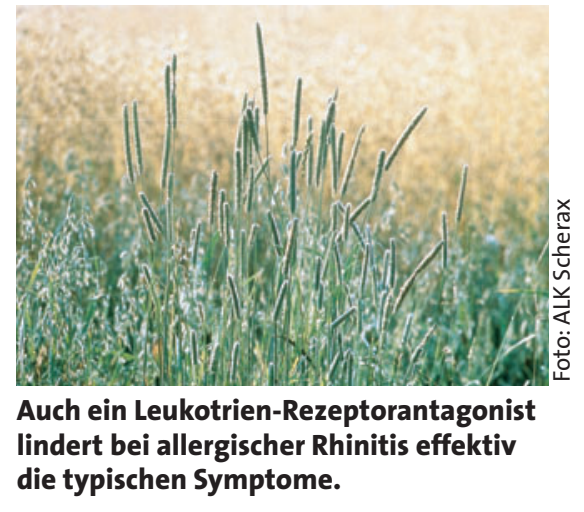

Fazit

In einer Dosierung von einmal täglich $10 \mathrm{mg}$ gegeben beeinflusst der Leukotrien-Rezeptorantagonist Montelukast die Symptomatik bei Patienten mit einer saisonalen allergischen Rhinitis vergleichbar effektiv wie das Antihistaminikum Loratadin. Empfehlenswert dürfte diese Medikation vor allem für solche Patienten sein, die gleichzeitig auch an Asthma leiden.

$b k$

Philip G et al. Montelukast for treating seasonal allergic rhinitis: a randomized, double-blind, placebo-controlled trial performed in the spring. Clin Exp Allergy 2002; 32: 1020-8

16 Jahren war dagegen ein Zusammenhang zwischen Provokationstest und Lungenfunktion, nicht aber den Eosinophilen oder NO zu erkennen. In beiden Gruppen ergab sich eine Assoziation von Eosinophilen und NO-Konzentration der Ausatemluft.

\section{Fazit}

Die bronchiale Hyperreagibilität ist bei kürzerer Krankheitsdauer eher mit der Atemwegsentzündung korreliert, während sie in späteren Stadien eher mit einer verschlechterten Lungenfunktion assoziiert ist. Diese Befunde zeigen erneut den durch die allergische Entzündung fortschreitenden RemodellingProzess beim chronischen Asthma. bk

Grönke L et al. The relationship between airway hyper-responsiveness, markers of inflammation and lung function depends on the duration of the asthmatic disease. Clin Exp Allergy 2002; 32: 57-63 